Revue d'histoire de l'Amérique française

Ali REVUE D.HISTOIRE DE L'AMÉRIQUE FRANÇAISE

\title{
La fabrication des caractères d'imprimerie à Montréal au milieu du XIX ${ }^{\mathrm{e}}$ siècle
}

\section{Bernard Dansereau}

Volume 47, numéro 1, été 1993

URI : https://id.erudit.org/iderudit/305183ar

DOI : https://doi.org/10.7202/305183ar

Aller au sommaire du numéro

\section{Éditeur(s)}

Institut d'histoire de l'Amérique française

\section{ISSN}

0035-2357 (imprimé)

1492-1383 (numérique)

Découvrir la revue

\section{Citer cette note}

Dansereau, B. (1993). La fabrication des caractères d'imprimerie à Montréal au milieu du XIX ${ }^{\mathrm{e}}$ siècle. Revue d'histoire de l'Amérique française, 47(1), 83-92. https://doi.org/10.7202/305183ar d'utilisation que vous pouvez consulter en ligne. 


\section{NOTE DE RECHERCHE}

\section{LA FABRICATION DES CARACTÈRES D'IMPRIMERIE À MONTRÉAL AU MILIEU DU XIX ${ }^{\mathrm{e}}$ SIÈCLE}

BERNARD DANSEREAU

L'industrie de la fabrication des caractères d'imprimerie a connu un véritable développement autonome au Québec au XIX ${ }^{\mathrm{e}}$ siècle, entre 1835 et 1897. Elle a permis aux imprimeurs québécois et canadiens de s'approvisionner au Québec. Plusieurs entreprises ont commercialisé des caractères importés des États-Unis ou de Grande-Bretagne. Une seule, cependant, les a produits à Montréal: la Montreal Type Foundry, à qui succédera en 1872, la Dominion Type Foundry. A partir de 1897, avec la vente de cette dernière à la Toronto Type Foundry, il ne se produira plus de caractères d'imprimerie au Canada car elle se concentre sur des activités de commercialisation de matériels d'imprimerie.

En janvier 1835, un article paru dans L'Ami du peuple, de l'ordre et des lois annonce l'ouverture d'une fonderie de caractères ${ }^{1}$ pour le début du mois de mai $1835^{2}$. L'initiative en revient à Thomas Guerin qui dit s'appuyer sur une longue expérience pour justifier son entreprise. La Fonderie de caractère d'imprimerie de Montréal (principalement connue sous le nom anglais de Montreal Type Foundry) sera la première fonderie de caractères à Montréal.

$\mathrm{Au}$ départ, Thomas Guerin requiert les services de Pierre-Édouard Leclère et de John Jones à titre d'agents. Ceux-ci n'en sont pas à leurs

1. Les sources relatives aux fonderies de caractères sont rares. À notre connaissance, il n'existe aucun fonds d'archives se rapportant à ces entreprises. Nous avons donc eu recours à des sources secondaires, ce qui nous a amené à privilégier l'analyse des fonderies de caractères comme industrie plutôt que d'étudier spécifiquement la Montreal Type Foundry.

2. L'Ami du peuple, de l'ordre et des lois, $1^{\text {er }}$ janvier 1835.

3. Le surintendant de police Pierre-Édouard Leclère et le marchand John Jones fondent, avec l'aide des Sulpiciens, en 1832, le périodique religieux L'Ami du peuple, de l'ordre et des lois.

RHAF, vol. 47, nº 1, été 1993 
premières armes dans le secteur de l'imprimerie ${ }^{3}$. Dès 1833 , ils sont les agents de la firme new-yorkaise Pell \& Brothers qui offre aux imprimeurs montréalais un large éventail de caractères d'imprimerie, de musique et autres articles susceptibles de les intéresser ${ }^{4}$, ainsi que ceux de la firme Robert Hoe and Company, le fabricant de presses à imprimer de New York.

L'entreprise de Thomas Guerin ouvre effectivement ses portes en mai 1835 et publie, dès la fin du mois, une liste de prix. Cette liste comporte un large éventail de caractères: du diamond au five line pica, des caractères spécialisés comme le scripte et le grec, ainsi qu'un choix de filets (lignes de différentes largeurs et longueurs) et d'ornements $^{5}$.

Les premières années de la nouvelle entreprise ont été difficiles car la conjoncture économique et politique ne se prête pas particulièrement à la venue d'une nouvelle industrie: crise agraire, baisse des exportations, crise politique qui culmine dans les insurrections de 1837-1838. Tout concourt à rendre périlleuse l'ouverture d'une nouvelle entreprise industrielle. La fermeture temporaire de certaines imprimeries, notamment celles de John Lovell et de Ludger Duvernay (La Minerve), restreint d'autant le marché de la fonderie de caractères.

Les entreprises manufacturières montréalaises de cette époque sont largement dépendantes du marché local ${ }^{6}$. Tout changement des conditions économiques et particulièrement dans l'industrie de l'imprimerie aura des conséquences considérables sur une entreprise comme la Montreal Type Foundry.

Il est vraisemblable de croire que les difficultés économiques forcent Thomas Guerin à fermer sa fonderie montréalaise pour tenter la même aventure à New York. En 1837, il achète tout le matériel de la fonderie de Hagar et Pell de New York après que celle-ci eût passé au feu. Une fonderie au nom de Thomas Guerin, sise au 57 rue Gold, figure dans les annuaires de la ville de New York de 1839 et 1840. Mais il n'est pas établi que cette entreprise ait vraiment fabriqué des caractères ${ }^{7}$.

4. L'Ami du peuple, de l'ordre et des lois, 14 juillet 1833.

5. L'Ami du peuple, de l'ordre et des lois, 16 mai 1835 et The Gazette, 16 mai 1835.

6. G. Tulchinsky, The River Barons: Montreal, Businessmen and the Growth of Industries and Transport, 1837-53 (Toronto, University of Toronto Press, 1977), 219. «Until near the end of the thirties, the city's manufacturing was small in scale and dependent mainly upon a very localized market.»

7. Stephen O. Saxe, «The Type Founders of New York City, 1840-1900», Printing History, 2,1 (1980): 12. 
La fermeture de l'entreprise montréalaise semble se confirmer à la lecture du catalogue L'Imprimerie d'Augustin Côté et Compagnie de Québec publié en 1844. L'imprimeur y mentionne que tous ses caractères proviennent d'Europe:

(que les propriétaires) ont reçu une magnifique collection de caractères, simples et ornés, des goûts les plus nouveaux et les plus riches; de superbes vignettes et d'ornements de toute espèce...

Ce n'est donc pas exagérer d'avancer que, au moyen de cette presse et d'une pareille collection de caractères et d'ornements, qui leur vient, sans exception, de Londres et de Paris (et tout le matériel vient d'Europe), les propriétaires du Journal de Québec peuvent lutter avec les plus belles impressions de Paris et de Londres ${ }^{8}$.

Si la Montreal Type Foundry opérait à cette époque, ce catalogue aurait certainement contenu des caractères provenant de Montréal, or il n'en est rien.

Après ces années difficiles, la Montreal Type Foundry change de propriétaire. Charles Theodore Palsgrave l'acquiert en $1845^{9}$. Jusquelà, C. T. Palsgrave était représentant de l'Inland Marine Insurance Company. En annonçant l'acquisition de la firme, Charles Theodore Palsgrave indique qu'un travailleur expérimenté est engagé, vraisemblablement un fondeur. Il pourrait s'agir du même Thomas Guerin, puisque deux ans plus tard, Palsgrave annonce, dans La Minerve, que Thomas Guerin a définitivement quitté la Montreal Type Foundry ${ }^{10}$.

Les affaires de la compagnie prospèrent rapidement. Vers 1849 , C. T. Palsgrave établit une succursale de sa compagnie à Toronto, sous la direction de M. Freehan et qui porte le nom de la Toronto Type Foundry.

The convenience that the printers of Canada now have in being able to purchase at their own doors all they can require to furnish or renew an office will be apparent to all; while the TORONTO TYPE FOUNDRY, a branch of this establishment under the management of Mr. D. K. FREEHAN, Agent, Front street,

8. Épreuves de caractères de l'imprimerie d'Augustin Côté et Compagnie (Québec, Haute-ville, 1844).

9. The Gazette, 11 juin 1845.

10. Jean-Marie Lebel, Ludger Duvernay et La Minerve, étude d'une entreprise de presse montréalaise de la première moitié du XIXe siècle, mémoire de maîtrise (département d'histoire), Université Laval, décembre 1982, 60. 
Toronto, places the printers of each section of the province upon an equally favourable footing.
Montreal, 1851.
Charles T. Palsgrave ${ }^{11}$

La succursale torontoise est toujours en opération en $1857^{12}$ et même en $1872^{13}$. Ce qui laisserait croire que les activités torontoises de cette entreprise ont été plus longues que ne l'indique Elizabeth Hulze, qui signale que durant cette période, l'entreprise aurait cessé ses activités entre 1851 et $1861^{14}$.

C. T. Palsgrave entend publier rapidement un catalogue des caractères et ornements disponibles, ce qui démontre que la Montreal Type Foundry fondait effectivement des caractères à la fin des années 1840. Cependant, la parution de ce catalogue est retardée par le départ du surintendant de la compagnie. Le propriétaire a alors éprouvé de la difficulté à en embaucher un nouveau. Ce n'est finalement qu'en 1850 que la Montreal Type Foundry pourra produire son catalogue ${ }^{15}$.

La Minerve salue avec enthousiasme cette publication: «Cet ouvrage est de la plus belle exécution et n'en cède en rien à ceux qui nous viennent des fonderies américaines. Nous espérons que les imprimeurs accorderont à Mr. Palsgrave l'encouragement qu'il mérite $^{16} . »$ The Gazette à son tour louange la qualité du travail de la fonderie montréalaise: «We observe, however, by the specimen book now before us, that he has brought his work to perfection, the face of the type is as beautiful as that of English. Printers should support home manufactures especially when the article is so $\operatorname{good}^{17} . » \mathrm{La}$ qualité du travail de la fonderie est reconnue puisqu'elle reçoit un prix d'excellence à l'Exposition universelle de Londres de 1851 où $\mathrm{C}$. $\mathrm{T}$. Palsgrave avait présenté une caisse de caractères d'imprimerie. La fonderie montréalaise ne participe cependant pas à l'Exposition universelle de Paris de $1855^{18}$.

$\mathrm{Au}$ milieu de la décennie 1840, Montréal est la plus importante ville du Canada, avec ses 50000 habitants. De nombreuses impri-

11. Elizabeth Hulze, A Dictionary of Toronto Printers, Publishers, Booksellers and the Allied Trades, 1798-1900 (Toronto, Anson-Carthwright Editions), 180.

12. Lovell's Canada Directory, 1857, 1244.

13. Starke's Pocket Almanac and General Register for 1872 (Montreal, Starke, 1872), 213.

14. Elizabeth Hulze, op. cit., 179.

15. Specimens of Printing Types and Ornaments cast at the Montreal Type Foundry (Montreal, C. T. Palsgrave, propriétaire).

16. La Minerve, 29 août 1850.

17. The Gazette, 28 août 1850.

18. Le Canada et l'exposition universelle de 1855 (Toronto, J. Lovell, 1856). 
meries y fonctionnent. On dénombre seize libraires, quinze imprimeurs, deux graveurs et lithographes. Une décennie plus tard, les libraires sont au nombre de vingt-sept, les imprimeurs de quatorze et les graveurs et lithographes de sept. L'entreprise a donc accès à un marché important, surtout si on y ajoute les imprimeries de Québec et des autres villes de la province.

À cette époque, la fabrication des caractères d'imprimerie est un processus totalement artisanal, long et complexe. La première étape de la création du caractère est confiée à un artiste qui doit dessiner l'ensemble des caractères de la police, soit de 100 à 200 signes différents. L'artiste doit aussi décliner ces signes dans les différentes forces corps car il n'est pas possible d'utiliser le même dessin pour toutes les grosseurs de corps. Pendant des décennies, ces règles de création se sont transmises par tradition orale.

L'étape suivante consiste à graver les poinçons et les contrepoinçons qui serviront à la confection des matrices. Un artisan, très qualifié, reproduit les dessins des caractères sur un bloc métallique: le poinçon. Celui-ci terminé, l'artisan produit la matrice, constituée d'une pièce métallique où le dessin du caractère est gravé en creux. C'est à partir de la matrice que va se faire la fonte des caractères.

Vient ensuite la fabrication du caractère d'imprimerie. Dans les ateliers, où la production est manuelle, les ouvriers tiennent dans leur main gauche un moule; ils vont de la main droite puiser dans une chaudière du métal en fusion avec une petite cuiller. Ensuite, simultanément avec un mouvement de haut en bas de la main gauche, ils versent le métal dans le moule. Ils soulèvent ensuite brusquement le moule pour faire rapidement descendre le métal, avant qu'il ne durcisse. Finalement, ils ouvrent le moule et laissent s'échapper le caractère terminé. Le métal utilisé pour la fonte est ordinairement un alliage d'étain, de plomb et d'antimoine, produit sur place dans des proportions variant d'une fonderie à l'autre.

Mais voilà qu'au cours des années 1830 , le fondeur new-yorkais David Bruce $\mathrm{J}^{\mathrm{r} 19}$ arrive à fabriquer la première machine à produire des

19. Le père et l'oncle de David Bruce, natifs d'Écosse, ouvrent une imprimerie à New York en 1806. Elle est, en 1809, la plus grosse imprimerie de cette ville. En 1813, les frères Bruce introduisent la stéréotypie dans leur entreprise. Craignant ne plus pouvoir s'approvisionner en caractères, ils acquièrent une participation dans la Starr Foundry. Leur succès dans la fonte de caractères est si rapide que, dès 1816 , ils se départissent de l'imprimerie pour ne conserver que la fonderie de caractères. Après le retrait de David Bruce en 1822, George Bruce continue les opérations de la fonderie, s'associant avec David Bruce Jr, le fils de David. David Bruce Jr est à la fois un fondeur de caractères et un graveur au poinçon. Au milieu des années 1830, il quitte l'entreprise de fonderie pour travailler sur une machine à mouler les caractères. Sa première machine est produite en 1838. Quelques années plus tard, elle sera en opération dans la majeure partie des fonderies américaines et européennes. 
caractères un à un et sans arrêt, qui soit vraiment efficace. Celle-ci permet de produire les petits et les gros caractères, ainsi que les filets. Cependant, les divers ornements restent fabriqués à la main jusqu'à l'invention d'une autre machine par Henry Barth de la Cincinnati Type Foundry, en 1888. L'invention de Bruce est l'aboutissement de recherches très anciennes et son introduction dans l'industrie constitue un immense progrès tant par la rapidité d'exécution que par la qualité du produit.

Dès 1856, la Montreal Type Foundry, la première à Montréal ${ }^{20}$, possède douze de ces machines, vraisemblablement celles-là mêmes que David Bruce a dessinées ${ }^{21}$. Il est possible que l'installation en ait été faite par le machiniste William Berry.

Nos sources permettent de brosser un portrait de l'entreprise et décrire le fonctionnement des machines ${ }^{22}$. L'entreprise est divisée en plusieurs ateliers qui correspondent chacun à une tâche et à une fonction spécifique exécutée par des travailleurs et des travailleuses. À chaque atelier correspond un nombre spécifique d'entre eux répartis proportionnellement au temps nécessaire à la réalisation de chacune des opérations.

L'entreprise possède, en 1864, neuf ou dix machines à fondre les caractères. Chacune d'elles est opérée par un seul travailleur, «qui peut fabriquer les plus petites pièces, jusqu'à 200 d'entre elles, en l'espace d'une minute ${ }^{23}$ »"

Voyons comment l'opération se déroule. Au sommet de la machine se trouve un récipient qui contient du métal en fusion. À l'intérieur du récipient, un piston pousse une certaine quantité de métal à l'intérieur d'une ouverture. L'ouvrier, à l'aide d'une manivelle, insère le métal contenu dans l'ouverture du moule où se trouve la matrice du caractère à reproduire. Une fois le métal engagé, le caractère est produit presque instantanément et, aussitôt refroidi, il est expulsé du moule vers une glissade inclinée, au bas de laquelle se ramassent les caractères terminés. Le travail se fait à un rythme d'environ 200 caractères à la minute. La fonte des caractères est la

20. Peter Bishoff, Les ouvriers mouleurs à Montréal: 1859-1881, mémoire de maîtrise (histoire), Université du Québec à Montréal, 1986, 54.

21. Grand Trunk Railway, General Railroad Celebration Committee, Montreal in 1856: a Sketch Prepared for the Celebration of the Opening of the Grand Trunk Railway of Canada (Montreal, J. Lovell, 1856).

22. The Gazette, 20 juillet 1864; Canada Railway Advertizing Company, Montreal Business Sketches with a Description of the city of Montreal, its public Buildings and Places of interest and the Grand Trunk Works at Point St. Charles, Victoria Bridge, etc, etc. (Montreal, Printed by M. Longmore \& Co. Gazette Printing, 1864).

23. The Gazette, 20 juillet 1864. 
seule opération effectuée par une machine. Toutes les autres, soit à la préparation du travail ou à la finition du caractère, restent essentiellement manuelles.

La préparation du métal est faite au dernier étage de l'immeuble. Les ouvriers responsables mélangent le plomb, l'antimoine et l'étain nécessaires aux caractères de bonne qualité. La proportion des différents métaux oscille autour de 75 pour cent pour le plomb, 20 pour cent pour l'antimoine et 5 pour cent pour l'étain. Une certaine quantité de bronze ou de cuivre peut aussi être ajoutée ${ }^{24}$. Une fois le mélange terminé, l'alliage est descendu à l'atelier de fabrication proprement dit, où se trouvent les machines.

Même fondu et refroidi, le caractère est encore muni d'un petit bout de métal, provenant de son passage dans le moule. La tâche de l'éliminer est confiée à de jeunes garçons. Les caractères sont ensuite envoyés à de jeunes filles qui doivent les polir. Ce travail demande, selon The Gazette, une grande dextérité: «This process is very interesting to witness, the dexterity with which the operation is performed being really wonderful.» ${ }^{25}$

Dernière étape, un ouvrier qualifié verra à examiner les caractères, à éliminer les défectueux et enfin à les préparer pour la livraison.

La description de l'ensemble des opérations permet de comprendre tout le processus de fabrication et de voir la spécialisation du travail qui règne dans un tel atelier. Nous nous trouvons devant la forme la plus simple de la coopération à l'intérieur de la manufacture. L'arrivée des machines ne modifie en rien le principe de cette coopération qui existait à l'époque où toute la production était manuelle. Elle change, tout au plus, le rapport entre le nombre d'ouvriers fondeurs et le nombre des personnes affectées aux autres tâches: préparation du métal, cassage et frottage des caractères, etc.

Les machines utilisées à la Montreal Type Foundry sont fabriquées à l'usine même. Cette tâche est confiée à M. Drummond, dont l'atelier est situé au troisième étage de l'édifice. Il succède vraisemblablement à W. Berry qui a travaillé quelque temps à la fonderie. $L a$ Gazette ne précise toutefois pas si les machines sont véritablement produites ou simplement assemblées, avec des pièces fabriquées ailleurs. La fonderie garde précieusement dans un coffre-fort plus de

24. La Montreal Type Foundry utilise, en $1861,76,7$ pour cent de plomb, 13,5 pour cent d'antimoine, 5,9 pour cent d'étain et 3,9 pour cent de bronze. En 1871, la proportion varie quelque peu: 71,6 pour cent de plomb, 22,7 pour cent d'antimoine, 1,4 pour cent d'étain et 1,4 pour cent de cuivre qui remplace le bronze. Ces données ont été compilées à partir des recensements nominaux de 1861 et 1871 .

25. The Gazette, 20 juillet 1864 . 
10000 matrices représentant un des actifs les plus précieux de l'entreprise, compte tenu de l'effort et du temps qu'il faut consacrer à leur production ${ }^{26}$.

Sur le même étage se trouve le département de stéréotypie. Le stéréotype ou cliché est un moulage de la forme imprimante qui permet de remplacer les caractères typographiques pour l'impression de l'imprimé. Ce procédé permet à l'imprimeur de prévenir l'usure prématurée des caractères tout en lui offrant la possibilité d'accélérer l'impression par l'emploi de multiples clichés. Le cliché de forme plate ou semi-cylindrique peut être monté sur la presse à platine, la presse cylindrique ou la rotative. Il est fréquent, à cette époque, de voir cohabiter dans la même entreprise la fabrication des caractères d'imprimerie avec un atelier de stéréotypie. La raison principale tient au fait que les deux activités de production ont plusieurs points communs, dont l'utilisation du métal en fusion comme élément principal du travail.

$\mathrm{Au}$ second étage se trouvent les bureaux de la direction et l'entrepôt où, en plus des caractères prêts pour la livraison, on remise tout ce qui est nécessaire à l'imprimeur: encres, presses, etc. Car en plus de fondre des caractères, des filets et des ornements, l'entreprise offre à ses clients les caractères provenant d'autres fonderies, notamment ceux de la fonderie L. Johnson \& Company de Philadelphie. Elle sert aussi d'agent pour le fabricant de presses à imprimer new-yorkais, Robert Hoe et $\mathrm{Co}$, et pour le fabricant d'encres, lui aussi new-yorkais, George Mather ${ }^{27}$.

Il est possible qu'une autre fonderie de caractères ait existé à Montréal vers 1855 . Son initiateur serait nul autre que Thomas Guerin, fondateur de la Montreal Type Foundry en 1835. On retrouve un Thomas Guerin, fondeur, dans le Montreal Directory de $1847^{28}$, année où Thomas Guerin aurait quitté, pour la seconde fois, la Montreal Type Foundry ${ }^{29}$. En 1855, la Canada Type Foundry est aussi connue sous le nom de Guerin \& Company ${ }^{30}$.

Aucune référence à cette dernière entreprise n'existe après 1855 . Rien n'indique cependant que cette compagnie ait effectivement vendu ni encore moins produit des caractères d'imprimerie à Montréal.

\footnotetext{
26. Canada Railway Advertising Company, Montreal Business Sketches..., op. cit.

27. Elizabeth Hulze, op. cit., 180 et Canada Directory (1857), 1244.

28. R. W. S. Mackay, The Montreal Directory for 1847, 91.

29. Jean-Marie Lebel, op. cit., 60.

30. R. W. S. Mackay, The Montreal Directory for 1855, 104 et 364.
} 
Dans un article où il relate ses souvenirs, Peter A. Crossby, membre fondateur de la Montreal Typographical Union et plus tard gérant de la Dominion Type Foundry, mentionne l'existence de la Guerin's Type Foundry qui, d'après lui, serait devenue par la suite la Palsgrave $^{31}$. Nous savons que ce ne fut pas le cas. Peut-être Crossby fait-il référence à la première entreprise de Thomas Guerin achetée par C. T. Palsgrave.

Au début des années 1870, la Montreal Type Foundry vit des moments difficiles. Une réorganisation complète s'opère alors. C. T. Palsgrave s'associe avec un fondeur du nom de W. G. Stethem. Ce dernier aurait détenu une entreprise pendant quelque temps sous la raison sociale de Stethem's Type Foundry and Printers Agency sur la rue Saint-Jacques ${ }^{32}$. Durant l'année 1872, l'association de Palsgrave et Stethem donne naissance à la Palsgrave et Stethem Type Foundry, qui opère aussi sous le nom de Montreal Type Foundry ${ }^{33}$.

Cette réorganisation ne peut empêcher la faillite ${ }^{34}$. À l'été 1873 , un groupe d'actionnaires prend la relève et dépose une demande d'incorporation auprès du gouvernement fédéral pour une nouvelle firme, la Dominion Type Foundry. Parmi les actionnaires, plusieurs œuvrent dans le secteur de l'imprimerie. C'est notamment le cas des frères Thomas et Richard White, propriétaires de The Gazette ainsi que de l'éditeur et imprimeur George-Édouard Desbarats. Au total vingt-neuf personnes figurent sur la demande de lettres patentes.

Le gouvernement fédéral octroie les lettres patentes le 14 octobre 1873. Les premiers directeurs de la nouvelle compagnie sont les éditeurs Richard White (The Gazette) et George-Édouard Desbarats (Canadian Illustrated News et L'Opinion Publique), le papetier Alexander Buntin (Valleyfield Paper Company), le banquier torontois John McDonnell et le marchand montréalais Daniel J. Rees (D. Rees and Company). C. T. Palsgrave n'apparaît pas à ce moment au conseil d'administration, mais va y venir quelques années plus tard.

La Dominion Type Foundry poursuit le travail de la Montreal Type Foundry, offrant une gamme variée d'articles pour les imprimeurs. En plus de fondre elle-même ses caractères, elle détient l'exclusivité de la vente de nombreux produits, notamment des caractères de plusieurs fonderies américaines: soit ceux de Johnson Type

31. Printer and Publisher, avril 1900, 6.

32. W. J. Patterson, Statement Relating to the Home and Foreign Trade of the Dominion of Canada: Also, Annual Report of the Commerce of Montreal for 1872 (Montreal, 1872), 129.

33. Starke's Pocket Almanac and General Register for 1873 (Montreal, Starke, 1873), 213.

34. La Minerve, 7 avril 1873; The Gazette, 4 avril 1873. 
Foundry, Geo. Bruce's and Son and Company, James Connor's Son, Farmer and Little and Company. Elle offre aussi les encres de Chas. Johnson and Company, de Celebrated Book and Colored Inks, de Geo and Monell's New Inks ainsi que les presses de Robert Hoe and Company, de C. Potter Jr and Company, de G. P. Gordon and Company, de Campbell Printing Press Company et de Globe Manufacturing Company, etc. Au cours des années, d'autres fabricants viendront s'ajouter à cette liste.

À la fin du siècle, la crise économique, qui entraîne un ralentissement des activités, la forte concurrence américaine mais surtout l'arrivée des machines à composer vont avoir des conséquences désastreuses sur l'industrie de la fonte des caractères. Les machines à composer permettent de produire plus rapidement et à un moindre coût. L'avènement de ces dernières fait perdre de nombreux clients à la Dominion Type Foundry, notamment parmi les imprimeries de journaux qui, dès 1891, suivent la tendance nord-américaine et s'équipent de ces nouvelles machines. Or les journaux représentent la plus grande partie du marché des caractères d'imprimerie. Cette baisse de la clientèle entraîne la faillite de la Dominion Type Foundry qui est achetée par une entreprise ontarienne, en 1897, la Toronto Type Foundry. Celle-ci suspend la production à Montréal, se contentant d'en faire un point de vente de matériel d'imprimerie, notamment de caractères.

Cet épisode clôt définitivement l'histoire de la production de caractères d'imprimerie à Montréal, mais aussi au Canada. Montréal aura abrité, en effet, la seule entreprise canadienne de fonte de caractères d'imprimerie. 